\title{
Speckle-displacement-based wavemeter for mode-hop and side-mode detection
}

\author{
Jamal, Muhammad T.; Jakobsen, Michael L.; Hanson, Steen G.; Hansen, Anders K.; Jensen, Ole B.
}

\section{Published in:}

Applied Optics

Link to article, DOI:

10.1364/AO.445383

Publication date:

2022

Document Version

Peer reviewed version

Link back to DTU Orbit

Citation (APA):

Jamal, M. T., Jakobsen, M. L., Hanson, S. G., Hansen, A. K., \& Jensen, O. B. (2022). Speckle-displacementbased wavemeter for mode-hop and side-mode detection. Applied Optics, 61(4), 989-994.

https://doi.org/10.1364/AO.445383

\section{General rights}

Copyright and moral rights for the publications made accessible in the public portal are retained by the authors and/or other copyright owners and it is a condition of accessing publications that users recognise and abide by the legal requirements associated with these rights.

- Users may download and print one copy of any publication from the public portal for the purpose of private study or research.

- You may not further distribute the material or use it for any profit-making activity or commercial gain

- You may freely distribute the URL identifying the publication in the public portal

If you believe that this document breaches copyright please contact us providing details, and we will remove access to the work immediately and investigate your claim 


\title{
Speckle-displacement-based wavemeter for mode-hop and side-mode detection
}

\author{
Muhammad T. Jamal, ${ }^{1}$ Michael L. Jakobsen, ${ }^{2 *}$ Steen G. Hanson, ${ }^{2}$ \\ Anders K. HAnsen, ${ }^{2}$ Ole B. Jensen, ${ }^{2}$ \\ ${ }^{I}$ Department of Health Technology, Technical University of Denmark, Ørsteds Plads, 2800 Kgs. \\ Lyngby, Denmark \\ ${ }^{2}$ Department of Photonics Engineering, Technical University of Denmark, Frederiksborgvej 399, 4000 \\ Roskilde, Denmark \\ *mlja@fotonik.dtu.dk
}

\begin{abstract}
A speckle-displacement-based wavemeter is combined with a spatial-fundamentalmode-pass filter to eliminate the influence of multi-mode operation on the directionality of the resulting output from a Distributed Bragg Reflector tapered Laser (DBR laser). The proposed setup is characterized theoretically and experimentally, and detections of modehops and side-mode suppression ratio in the optical output are demonstrated. The laser illuminates a rough surface at an oblique angle, and a camera observes the corresponding speckle pattern from an almost identical back-scattering direction. As the wavelength of the laser shifts, the speckle pattern responds with a corresponding displacement, which is approximately linear with respect to the shift within the detection area. The wavemeter tracks continuously the shifts of the speckles pattern by tracking the peak of the covariance function of sequentially acquired images. In this way, the speckle-displacement-based wavemeter achieves a spectral resolution of $10.4 \mathrm{MHz}$. Mode-hops in the laser do not cause any impeding decorrelation of the speckle patterns. Interestingly, the actual side-mode suppression ratio is related to the peak height and width of the absolute covariance function. A wavemeter, which is capable of measuring wavelength, mode-hops and side-mode suppression ratio, is highly useful for spectroscopy, quantum optics, nonlinear frequency conversion and other applications requiring stable single-frequency laser light, especially when using diode lasers.
\end{abstract}

(C) 2021 Optica Publishing Group under the terms of the Optica Publishing Group Open Access Publishing Agreement

\section{Introduction}

In many $\mathrm{CW}$ laser applications, it is critically important to ensure stable, single frequency operation of the laser source. In particular, spectroscopy and quantum optics applications require wavelength stability of the order of a fraction of the line width, typically in the order of MHz. Other applications such as CW nonlinear frequency conversion in long crystals [1] [2], is also highly wavelength-dependent. An important detail about some lasers such as diode lasers is that they may not always lase in single-frequency operation, but may perform modehops or lase in a multi-mode operation. Therefore, the ideal wavemeter should be capable of determining not only the center wavelength with very fast measurement speed and high refresh rate, but also the side-mode suppression ratio.

During the last decade, a series of spectrometers and wavemeters have emerged based on random speckle patterns as indicators for either the absolute wavelength or a change in wavelength. Speckles arise when a radiated field from a source or a scattering medium has sufficient coherence and spatial variation in its phase front. After sufficient propagation, interference gives rise to high variance in the spatial intensity distribution [3][4][5]. This results in a fine structure of the speckles equal to the resolution limit of the optical setup. To be more precise, all speckle-based wavemeters or spectrometers depend on the recognition of 
changes in a wavelength-dependent speckle pattern. Consequently, this calls for the smallest granular structures in the speckle pattern to provide for the highest resolution.

Speckle-based wavemeters/spectrometers rely on speckle dynamics in two differing ways: either by probing the erratic change in a speckle pattern, i.e. speckle decorrelation, or by using a linear displacement of a rather rigid speckle pattern. A good overview of the various implementations have been given by Cao.[6]

The majority of speckle-based spectrometers relying on speckle decorrelation take advantage of the scrambling of the incident coherent field in a media. Some instances include a tapered fiber [7] giving an absolute spectral resolution of $40 \mathrm{pm}$; a disordered photonic chip with a spectral resolution of $750 \mathrm{pm}$; a single mode fiber using Rayleigh scattering [8] to achieve a resolution of 6 femtometer with a $100 \mathrm{~m}$ fiber; an integrating sphere [9] giving subfemtometer wavelength resolution.

Common for the above implementations is the necessity of establishing a look-up table for any sufficiently distinct speckle pattern that may occur throughout the applied range of wavelengths. A change in wavelength gives rise to a speckle decorrelation, and the look-up table must be sufficiently closely sampled so that any wavelength within the given range can be recognized. Further, for all the above implementations the resolution is given by the effective optical path length differences, being it in a fiber, in a disordered photonic chip or in an integrating sphere.

The speckle-based wavemeter (SW) concept discussed here, relies on using speckle displacement as a tool for probing wavelength changes in a coherent source. The setup, reported previously, showed a resolution of $100 \mathrm{MHz}$ at a wavelength of $760 \mathrm{~nm}$, being equal to a wavelength resolution of $200 \mathrm{fm}$ [10]. A competitive resolution was not achieved as compared with the implementations mentioned above. However, the setup was simple, as it only involved a scattering surface, here a sandblasted aluminum plate, and a CMOS array. A way in which wavelength changes can be followed on a fast time scale is by shearing the speckle pattern [11] across a grating-like structure [12]. No intermediate optical components were required. Besides, a linear dependency between the wavelength shift and the speckle displacement was shown theoretically and experimentally.

In the present paper, we will show a ten-fold increase in resolution confirmed by simultaneous measurements with a scanning Fabry-Perot Interferometer (sFPI). The theory is supported by concurrent SFPI and SW measurements from a tapered laser diode that emits its main line with possible side modes. We show that the combination of a spatial filter and the speckle-based wavemeter is capable of detecting reductions in side-mode-suppression or even mode-hops in the laser source. The theory shows that the correlation width and correlation amplitude in the SW system reveals the strength of the sidebands. A summary will give a short overview of the findings and the possibilities for further improvements.

\section{Theory}

Fig.1 shows the basic setup for probing wavelength changes of a beam incident onto a diffuse surface. The incident beam will be described as a Gaussian scalar field $U[\mathbf{r}]$. At incidence, the field is assumed to be a plane wave with a wavelength of $\lambda$ and a $1 / \mathrm{e}$ radius of $w_{0}$ (note $1 / \mathrm{e}^{2}$ for intensity).

The wavenumber $k$ of the incident field is defined as the length of the propagation vector of the field, $k=\left|\mathbf{k}_{1}\right|=2 \pi / \lambda$

$$
k=k_{0}-\Delta k / 2 \text { to } k=k_{0}+\Delta k / 2
$$

incidence of $\alpha_{1}$ relative to the normal $\hat{\mathbf{n}}$

$\mathbf{k}_{1}$ remains unchanged at an angle of 
the diffuser is observed in the 2D p-coordinate system at a distance of $L$, and at an angle of $\alpha_{2}$ relative to the normal of the surface. We assume that the setup is mechanically static, thus the length of the wavenumber $\left|\mathbf{k}_{2}\right|$ of the detected scattered field is identical to the length of the wavenumber of the incident field, $k=\left|\mathbf{k}_{2}\right|=\left|\mathbf{k}_{1}\right|$.

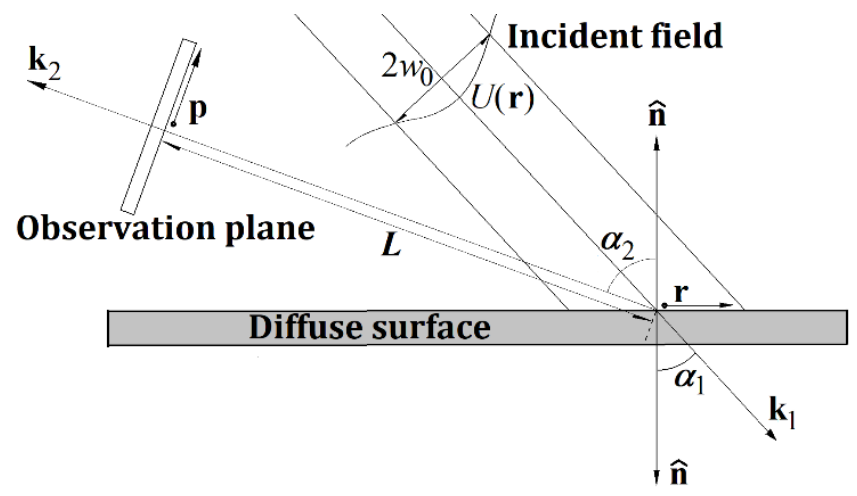

Fig.1. The schematics illustrate the configuration. The scattered and observed fields are described in the 2D r-and p-coordinate systems, respectively. Any relative distance in the observation plane is described by $\Delta \mathbf{p}$.

The incident Gaussian field is scattered by an entirely imaginary phase function, $\Psi[\mathbf{r}]$, which is presented by a Gaussian stochastic process. We assume that phase function is deltacorrelated [10]:

$$
\left\langle\Psi\left(\mathbf{r}_{1}\right) \cdot \Psi *\left(\mathbf{r}_{2}\right)\right\rangle=\frac{4 \pi}{k^{2}} \cdot \delta\left(\mathbf{r}_{1}-\mathbf{r}_{2}\right)
$$

The scattered field is propagated to the observation plane, using the Fresnel diffraction theorem, and here the intensity $I[\mathbf{p} ; k]$ can be determined for the two wavenumbers. However, due to the stochastic nature of the fields and their intensities, the cross covariance of the intensity observed before and after the change in wavenumber is preferred:

$$
\begin{aligned}
C\left(\mathbf{p}, \Delta \mathbf{p} ; k_{0}, \Delta k\right)= & \left\langle I\left(\mathbf{p}-\frac{\Delta \mathbf{p}}{2} ; k_{0}-\frac{\Delta k}{2}\right) I\left(\mathbf{p}+\frac{\Delta \mathbf{p}}{2} ; k_{0}+\frac{\Delta k}{2}\right)\right\rangle \\
& -\left\langle I\left(\mathbf{p}-\frac{\Delta \mathbf{p}}{2} ; k_{0}-\frac{\Delta k}{2}\right)\right\rangle\left\langle I\left(\mathbf{p}+\frac{\Delta \mathbf{p}}{2} ; k_{0}+\frac{\Delta k}{2}\right)\right\rangle,
\end{aligned}
$$

where $\Delta \mathbf{p}$ describes any relative distances in the observation plane, and is the variable in the covariance plane. As we shall see, the cross covariance function provides necessary statistical information about the speckle dynamics and its responses to changes in wavenumber.

In the case, the optical configuration is free space propagation from the input plane to the observation plane, we derive in [10] [12] the following expression for the cross-covariance function:

$$
\begin{gathered}
C\left(\mathbf{0}, \Delta \mathbf{p} ; k_{0}, \Delta k\right) \propto \\
\exp \left[-4 \frac{\left(\beta L \Delta k-k_{0} \Delta p_{x} \cos \left[\alpha_{2}\right]\right)^{2} \cos \left[\alpha_{1}\right]^{2} w_{0}^{2}}{16 L^{2} \cos \left[\alpha_{1}\right]^{4}+\Delta k^{2} w_{0}^{4}}\right]
\end{gathered}
$$


where $\beta \equiv \sin \left[\alpha_{1}\right]+\sin \left[\alpha_{2}\right]$. The cross-covariance function can be applied to entire speckle patterns e.g. obtained by a camera. In case a speckle displacement is present between the two images, the cross-covariance function will produce a distinct correlation peak, where its position from origin is a measure for the speckle displacement. The "gearing" between the speckle displacement $\Delta p_{x}$ and a given change in wavenumber $\Delta k$ is the most important parameter. The speckle displacement can be extracted from Eq.3 [10] [12] and it becomes:

$$
\Delta p_{x}=\frac{\beta L \Delta k}{k_{0} \cos \left[\alpha_{2}\right]} .
$$

As the changes in wavenumber increases the speckles will also decorrelate, and consequently the amplitude of the correlation peak drops. The decorrelation wavenumber $\Delta k_{d e c}$ is defined as the maximum change in wavenumber $\Delta k$ between two speckle images, where the correlation peak in the cross-covariance function still can provide a reliable measure for the speckle displacement. The decorrelation wavenumber $\Delta k_{\text {dec }}$ can be extracted from Eq.3 [12] as well. The decorrelation wavenumber is shaped as a Lorentzian function of $w_{0}$, with a width $\Delta k_{d e c}$ of:

$$
\Delta k_{\text {dec }}=\frac{4 L \cos \left[\alpha_{1}\right]^{2}}{w_{0}^{2}}
$$

The width of the correlation peak is a measure of the average speckle size $\rho_{0}$. The width of the correlation peak can also be extracted from Eq.3 [12] and becomes:

$$
\rho_{0}=\frac{2 L \cos \left[\alpha_{1}\right]}{k_{0} w_{0} \cos \left[\alpha_{2}\right]}
$$

The sensitivity in $\Delta k$ may be determined by the accuracy of finding the displacement of the speckle pattern, i.e. determining the peak position of the cross covariance for a large number of uncorrelated speckle patterns. As a rough estimate, the standard deviation of the position of the speckle displacement is proportional to the speckle size divided by the square root of the number of speckles on the CMOS array. This is in accordance with the results in a seminal paper on digital speckle correlation [13][14]. Please note that in case the speckle size approaches the pixel size, bias effects are likely to occur [14].

Assuming a uniform speckle displacement across the detector with area $A_{P D}$, the sensitivity in wavenumber $\Delta k_{\min }$ hence becomes:

$$
\Delta k_{\min } \propto \frac{4 L \cos \left[\alpha_{1}\right]^{2}}{k_{0} \beta \sqrt{A_{P D}} w_{0}^{2} \cos \left[\alpha_{2}\right]} .
$$

For practical application such as laser stabilization the speckle displacement will be only a fraction of the pixel size. For this purpose various ways to optimize the resolution in finding speckle displacement can be devised [15].

Generally, both stationary multiple modes, temporal shifts in mode structures and shifts in single mode positions during acquisition of samples will broaden the correlation peak and decrease its absolute peak value. Supplement 1 (Appendix A) derives the last situation where one mode shifts position according to a Gaussian stochastic process - exclusive mode hops during the acquisition time $T_{0}$. The variance of the Gaussian distribution is $\delta k_{0}$. The consequence is a broadening of the correlation peak determined from the acquired speckle patterns. Given the Gaussian distribution $P\left[k_{0}\right]$, the average gearing provides the same value as provided by Eq.4. However, the effective width of the correlation peak, which now 
includes both the original speckles size (Eq.6) and shifts in wavelength, is provided by Eq.A7:

$$
\rho=\frac{B \sqrt{2\left(1+\cos \left[\alpha_{1}\right]\right)+\beta^{2} w_{0}^{2} \delta k_{0}^{2}}}{w_{0} k_{0} \cos \left[\alpha_{2}\right]} .
$$

The height of the correlation peak, normalized to the height of a correlation peak for a stationary single mode, is provided by Eq.A8 as:

$$
A_{N}=\sqrt{\frac{4}{4+\beta^{2} w_{0}^{2} \delta k_{0}^{2} \sec \left[\alpha_{1}\right]^{2}}}
$$

Further, the decorrelation is provided by (Eq.A9) as:

$$
\delta k_{0} \square \frac{2}{\beta w_{0} \sec \left[\alpha_{1}\right]}
$$

\section{Experiment}

In Fig.2, the experimental setup is illustrated. The first optical component is a Distributed Bragg Reflector laser medium (DBR laser) that seeds a tapered laser medium [16]. Both laser media are located inside a shared cavity. This setup produces a nearly diffraction limited Gaussian beam $\left(M^{2}<1.5\right)$ with a high optical output $(1.2 \mathrm{~W})$ and at a wavelength of $1062 \mathrm{~nm}$. The seed laser commonly referred to as ridge-waveguide (RW) section is controlled with the diode current, $I_{R W}$, while the tapered amplifier (TA) is controlled with the diode current, $I_{T A}$. The seed laser is thermally stabilized/controlled with a Peltier element. The stability of the DBR tapered laser (DBR-TPL) is maintained by protecting it from optical feedback with the optical isolator of $\sim 60 \mathrm{~dB}$. A fraction $(5 \%)$ of the main beam is coupled out to the wavemeter. The speckle-based wavemeter (SW) consists of a spatial filter (pinhole of Ø40 $\mu \mathrm{m}+2$ lenses) inserted before the diffuser. The spatial filter eliminates any angular responses of the beam to transverse mode changes in the laser. At the diffuser, the incident beam radius is estimated to be $w_{0}=1.1 \mathrm{~mm}$. The diffuser is made of zirconia ceramics and replaces a diffuser made of aluminum, due to a lower thermal expansion coefficient $\left(5.7 \cdot 10^{-6} \mathrm{~m} / \mathrm{K} \mathrm{m}\right)$, in order to minimize any responses to thermal drift in the setup. The diffuser is orientated relative to the incoming beam at an angle of incidence of $\alpha_{1}=52.9^{\circ}$. At an observation angle of $\alpha_{2}=78.8^{\circ}$, and with a center-to-center distance of $L=9.3 \mathrm{~cm}$ a CMOS camera is mounted, facing the illuminated spot on the diffuser. The camera has $1280 \times 1024$ pixels of $5.2 \times 5.2 \mu \mathrm{m}$, and operates with a frame rate of $7.5 \mathrm{f} / \mathrm{s}$. Given the physical dimensions of the setup components and the speckles, this is the practical configurations with the highest $\alpha_{2}$-value achievable.

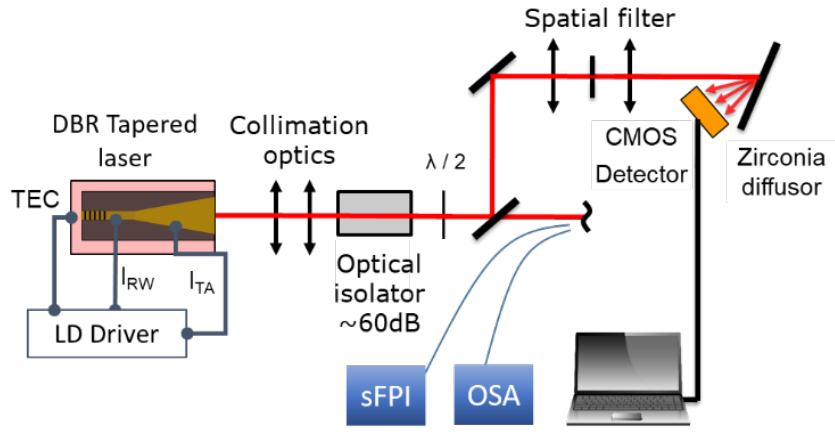


Fig.2. Schematic of the laboratory setup.

In the first experimental configuration, the SW is probing the output of the DBR laser in parallel with a commercial optical spectrum analyzer (OSA) (Advantest Q8347). The OSA returns the wavelength represented by the peak with the highest intensity in the acquired spectrum. The highest resolution of the OSA is $3.0 \mathrm{pm}$, and the sampling rate is $0.3 \mathrm{~S} / \mathrm{s}$. Further, the OSA returns a measure for the side-mode-suppression-ratio (SMSR) as well. The SMSR is specified as the intensity ratio (in $\mathrm{dB}$ ) between the second highest peak and the highest peak in the spectrum.

In order to observe even faster spectral fluctuations in the laser wavelength and for comparison of the SW performance, we used a scanning Fabry Perot interferometer (sFPI) (Thorlabs SA200-8B) in a second experimental configuration. The free spectral range (FSR) and resolution of the sFPI is $1.5 \mathrm{GHz}$ and $7.5 \mathrm{MHz}$, respectively. The sampling rate is 11 spectra per second or $11 \mathrm{~S} / \mathrm{s}$. The individual spectra from the sFPI often contain more than a single peak due to side-modes and/or multi-mode operation of the laser. Therefore, the weighted-average wavelength is determined for comparison with the output from the SW.

A continuous sequence of images are acquired and post processed in the following way: Initially, a centered 2D Hanning window is multiplied to all the raw images. Then, the spatial cross-covariance function between two images, $I_{1}$ and $I_{2}$, is implemented by first producing the spatial cross-power spectrum: $\operatorname{cr} P S=\mathfrak{I}\left\{I_{1}\right\} \times \mathfrak{I}\left\{I_{2}\right\}^{*}$, where $\mathfrak{I}\{\cdot\}$ is the discrete Fourier transform and * indicates the complex conjugate. In the cross-power spectrum, a Gaussian high-pass filter is implemented to remove the influence of low-frequency spatial structures in the speckle images. Often, these occur due to uneven illumination of the camera. Finally, the cross-covariance function $C(\Delta \mathbf{p})$ is completed by carrying out the inverse discrete Fourier transform to the spatial cross-power spectrum $C(\Delta \mathbf{p})=\mathfrak{J}^{I}\{c r P S\}-$ a consequence of the Wiener-Khinchin Theorem.

Initially, the correlation peak is located by searching for the maximum pixel value within the entire cross-covariance function, then the first and second moments are determined to center and reduce the area of interest, and finally, a Gaussian fit using least square fitting is applied to gain sub pixel precision.

Inserting the experimental parameters into Eq.6, the gearing can be estimated to $\Delta p_{x 0} / \Delta \lambda=$ 0.15 pixels $/ \mathrm{pm}$. From Eq.9, the typical speckle size becomes $\rho_{\mathrm{x}}=62.8 \mu \mathrm{m}$ (12.1 pixels), which agrees with an $\mathrm{e}^{-2}$-radius of the peak in the covariance function (acquired during single mode operation), which is measured to 11.3 pixels. From Eq.7, the decorrelation wavelength becomes $\Delta \lambda_{\text {dec }}=10.0 \mathrm{~nm}$.

The strategy for our data processing is to measure a shift of the speckle pattern as a response to a shift in wavelength. The wavelength of the laser can be manipulated by changing the temperature (TEC) of the seed laser, or by changing the diode current $\left(I_{R W}\right)$ to the seed laser. A long series of images are acquired for each experiment. Before processing a series of images, a reference image is selected, and all other images are correlated with this reference image. The reference images are located where the axis shows a shift of 0 pixels.

\section{Results}

In Fig.3, the SW and the OSA device probe the changes in wavelength as the diode current in the seed laser $\left(I_{R W}\right)$ is increased from $295 \mathrm{~mA}$ to $305 \mathrm{~mA}$ in 1000 steps of $10 \mu \mathrm{A}$. Each step takes $20 \mathrm{~s}$ to allow the laser to reach thermal equilibrium. For the SW, the image acquired at $I_{R W}=300 \mathrm{~mA}$ acts as the reference image for this series of images. A single mode hop occurs at $I_{R W}=298.6 \mathrm{~mA}$ in both plots. The digitization of the OSA device introduces three steps in the characteristics of $3 \mathrm{pm}$ per step. The noise equivalent wavelength of the SW is determined as the standard deviation of the linear part of the curve in Fig.3. The noise equivalent is estimated to 0.057 pixels. 


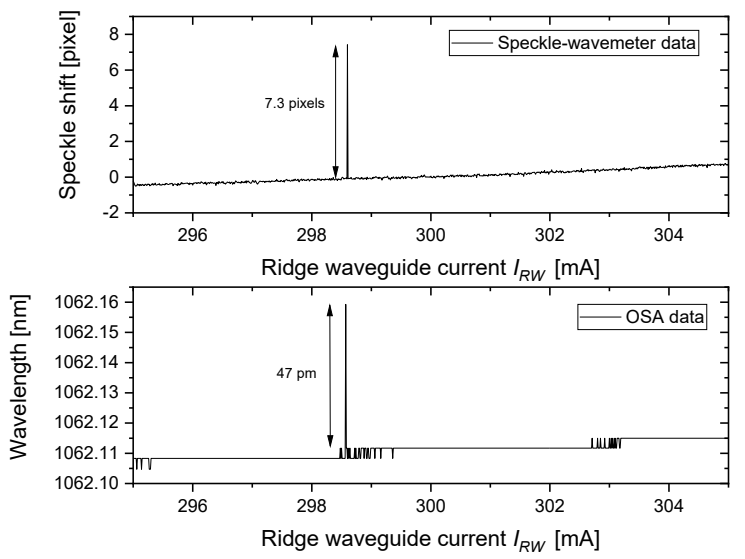

Fig.3. Simultaneous shifts of speckle pattern obtained with SW (top) and wavelength measurements with OSA (bottom) as a function of RW current.

Based on the slopes of the two curves (excluding the mode hop), the conversion factor between the SW and the OSA is estimated to be $7.0 \mathrm{pm} / \mathrm{pixel}(=1.86 \mathrm{GHz} / \mathrm{pixel}$, and in agreement with the result from Eq.6), thus the noise equivalent wavelength of the wavemeter measurement becomes $\Delta \lambda_{\min }=0.40 \mathrm{pm}(106 \mathrm{MHz})$ at maximum. Due to the relatively large time steps of $20 \mathrm{~s}$, this noise equivalent wavelength includes both slow variations $(\sim 20 \mathrm{~s})$ and fast variations in both laser and wavemeter operation.

In order to eliminate the slow variations, the $\mathrm{SW}$ is setup to measure in parallel with the sFPI, and at stable lasing parameters $\left(T=30.0^{\circ} \mathrm{C}\right.$ and $\left.I_{R W}=298.6 \mathrm{~mA}\right)$.

Fig.4 shows simultaneously frequency shifts measured with the sFPI and the SW. Here, the observed slow variation in the emission wavelength is due to the spectral instability of the DBR-TPL whereas the fast fluctuations constitute noise due to measurement limitation in addition to spectral noise of the DBR TPL. The root mean square deviation (RMSD) of the difference between the SW output and the sFPI measurements is $\Delta \lambda_{\min }=10.4 \mathrm{MHz}(0.0391$ $\mathrm{pm})$. Never the less, short term deviations in the SW frequency shift occur sporadically - in this case with up to $30 \mathrm{MHz}(0.113 \mathrm{pm})$ deviation from the sFPI data at $17 \mathrm{~s}$ to $25 \mathrm{~s}$.

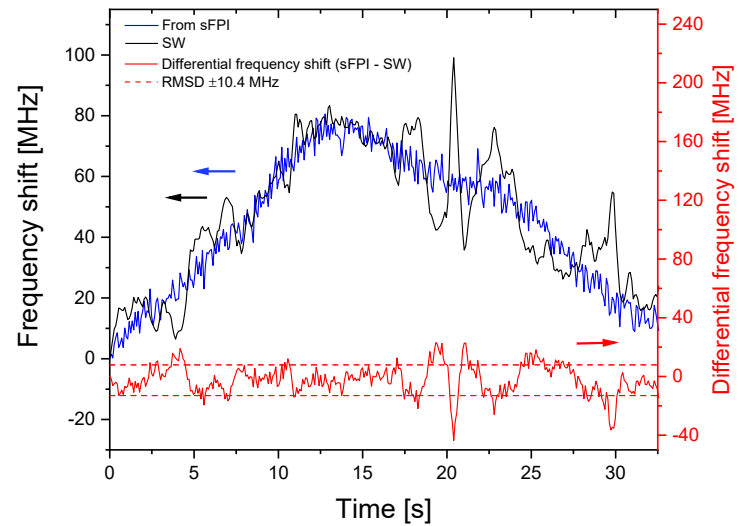

Fig.4. Frequency shift in the weighted-average emission frequency of the DBR-TPL obtained by the SFPI (blue) and the SW (black), obtained at full sample rates of the devices. The differential frequency $\left(f_{\mathrm{SPI}}-f_{\mathrm{SW}}\right)$ with root mean square deviation $\pm 10.4 \mathrm{MHz}$ is plotted in red. 
In order to demonstrate the response of the SW to various mode operations of the laser, the SW is setup to measure in parallel with the OSA. In this experiment, the temperature variations are used to vary the wavelength of the laser output - mostly due to the temperature dependence on the refractive index in the gain medium. The temperature changes provide a relative large variation in wavelengths, and therefore different mode constellations in the laser can be induced. This includes multimode operation, pure mode-hops and other instabilities in the laser operation.

Fig.5 illustrates the emission wavelength of the laser measured by using the OSA and the $\mathrm{SW}$ as a function of DBR-TPL operating temperature. Five events are marked with the vertical dashed lines. Three of these are mode hops and occur at the following temperatures: $T$ $=28.2^{\circ} \mathrm{C}$ (grey), $T=29.5^{\circ} \mathrm{C}$ (purple) and $T=30.15^{\circ} \mathrm{C}$ (green). At a temperature of $T=$ $29.5^{\circ} \mathrm{C}$, an extra and temporary mode hop is registered by the $\mathrm{SW}$,

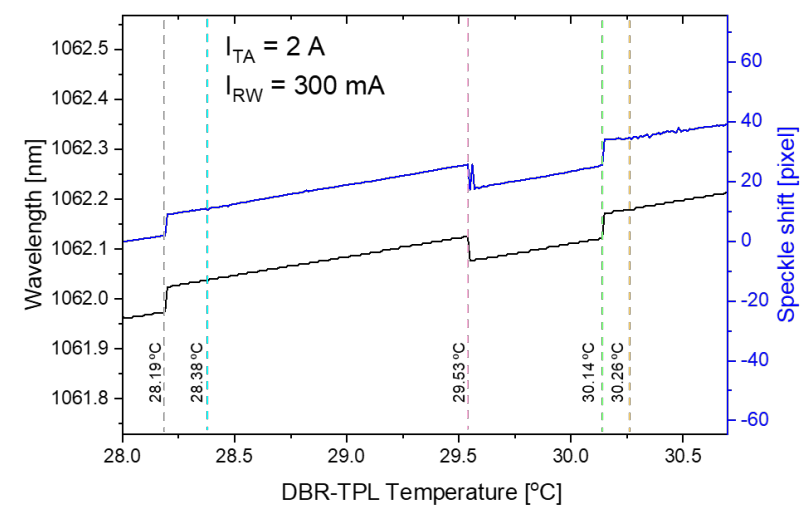

Fig.5. Emission wavelength measured by the OSA (black) and the SW (blue) as a function of DBR-TPL operating temperature.

Looking into more details of the correlation functions of the images, which are obtained during the experiments in Fig.5, we observe an interesting relationship between the FWHM of the autocorrelation peak and the side-mode-suppression-ratio (SMSR), measured by the OSA.

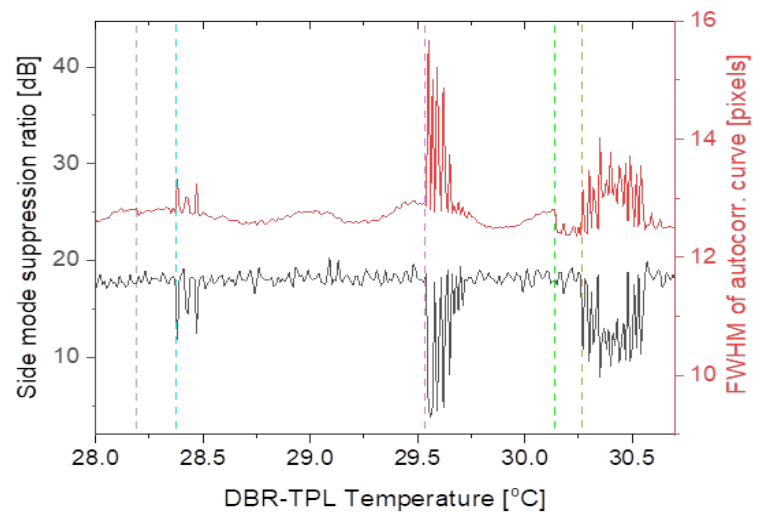

Fig.6. SMSR (black) of the DBR-TPL and FWHM (red) of the autocorrelation peak for speckle images.

In Fig.6, this relationship is illustrated as a function of the same horizontal axis (DBRTPL temperature interval). The FWHM of the autocorrelation peak increases proportional to 
the decrease in the SMSR of the laser. The low SMSR is an indication of multimode behavior of the laser, while high SMSR indicates single mode operation. Clearly, three events start at the following temperatures: $T=28.4^{\circ} \mathrm{C}$ (cyan), $T=29.5^{\circ} \mathrm{C}$ and $T=30.3^{\circ} \mathrm{C}$ (yellow) Interestingly, Fig.6 illustrates no response to the mode hop occurring at $T=28.2^{\circ} \mathrm{C}$, while Fig.5 illustrates no response to the drop in SMSR beginning at $T=28.4^{\circ} \mathrm{C}$ and $T=30.3^{\circ} \mathrm{C}$.

\section{Discussion}

The full spectra of the laser output, which are acquired by the OSA, are illustrated in Fig.7 as a function of the DBR-TPL temperature range.

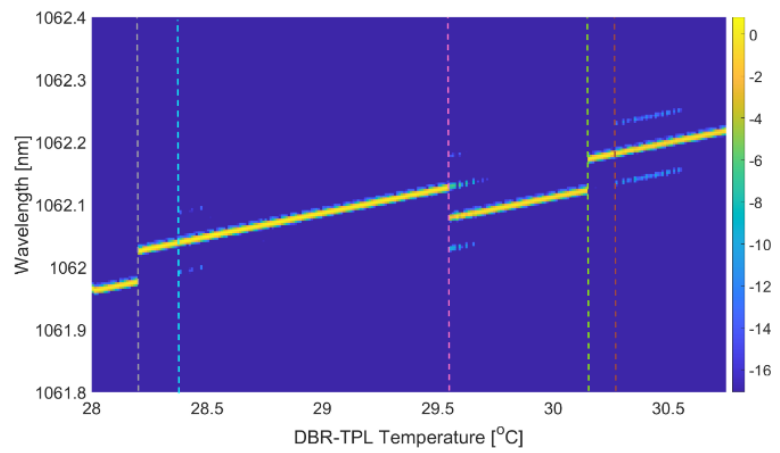

Fig.7. Emission spectra, acquired by the OSA, are plotted as a function of DBR-TPL operating temperature. The color bar indicates the normalized intensity in $\mathrm{dB}$

At the temperature of $T=28.2^{\circ} \mathrm{C}$, the mode hop appears to occur (almost) instantaneously and without appearance of multi-mode operation. For that reason, we expect no broadening of the correlation peak obtained by the SW. At the temperature of $T=28.4^{\circ} \mathrm{C}$, Fig. 7 illustrates multimode operation, but without mode hop. This is apparently, the reason for the slight broadening of the correlation peak occurring in Fig.6. At the temperature of $T=29.5^{\circ} \mathrm{C}$, Fig. 7 illustrates that the mode hop occurs during severe presence of multimode operation of the laser. Apparently, the laser switches between the original and the new wavelength, and probably inducing multimode operation as well. The SW has a sample rate 25 times faster than the OSA. Therefore, we expect the SW to acquire a single and temporary occurrence of the original wavelength, while the OSA has not. The multimode occurrence has not affected the position of the correlation peak, while the broadening of the peak is severe as it can be observed in Fig.6.

The broadening of the correlation peak is due to the appearance of temporal multimode behavior in the emission as it can be seen for the OSA in Fig.6. A theoretical investigation of the expected broadening is given in Supplement 1 (Appendix A), using the approximation in Eq.8 or Eq.A10. If we insert the relevant parameters for a setup and the observed broadening measured by the SW in Fig.6, we get a spectral broadening of $95 \mathrm{pm}$ at $29.6^{\circ} \mathrm{C}$. This is in fine agreement with the mode hop in Fig.7.

At the temperature of $T=30.15^{\circ} \mathrm{C}$, an instantaneous mode hop is observed by both the $\mathrm{SW}$ and the OSA. At a temperature of just $0.15^{\circ} \mathrm{C}$ higher, multimode operation occurs again. In this case, the multimode operation is biasing the measurements of the average correlation peak detected by the SW. The OSA detects the highest peak present in the acquired spectra, and therefore it measures the dominating wavelength unaffected. However, multimode operation is detected by both the SW and the OSA in Fig.6.

We expect that the low frequency variations in the FWHM of the autocorrelation in Fig.6 are measurements of the side-mode suppression beyond the resolution of the spectrum analyzer (OSA). 
In the present setup, the sample rate is determined by the update rate of the camera. However, if the camera is replaced/combined with spatial filtering velocimetry [11], a substantial increase in response time will be possible. This might reduce the sensitivity of the SW. However, it could enable fast response stabilization of laser systems, being sensitive to e.g. optical feedback.

In principle, the technique is not dependent on the optical wavelength. Only the sensitivity of the camera/detector arrangement defines the limits of the technique. However, as mentioned above, the decorrelation wavenumber $\Delta k_{d e c}$ sets a limit to the maximum change in wavenumber in between two images. However, in this case, we determined a value $\Delta k_{\text {dec }}=10$ $\mathrm{nm}$, which is far more than sufficient for e.g. stabilization of a laser diode. Typically, a reliable peak search in a cross-covariance function can be performed for normalized correlation values above levels of $15-20 \%$. Beyond $\Delta k_{\text {dec }}$, the correlation value drops below approximately $10 \%$, and the method will no longer provide reliable identifications of the wavelength. Further, the accuracy of measuring the peak location will drop as $\Delta k_{\text {dec }}$ increases.

\section{Conclusion}

This study demonstrates not only the improved sensitivity of the speckle-based wavemeter compared to its predecessor but also shows its capability to detect mode hops and multimode behavior of the laser. The spatial-fundamental-mode-pass filter is essential to maintaining the high resolution of the SW, while characterizing the laser outputs during both multi-mode operation and poor side-mode suppression. The zirconia diffuser provides a factor of four in less sensitivity towards thermal drift in the system compared with using aluminum - an effect we observed during the preliminary experiments. Improvements in the image processing allow SW to resolve shifts in the speckle pattern of less than a fraction of 1/200 of a pixel. Therefore, the sensitivity of the speckle wavemeter to wavelength shifts has been significantly improved to a resolution of $10.4 \mathrm{MHz}$.

Funding. European Commission (721766)

Acknowledgments. All the authors would like to thank Peter E. Andersen for his very valuable suggestions and feedback.

Disclosures. The authors declare no conflicts of interest.

Data availability. Data underlying the results presented in this paper are not publicly available at this time but may be obtained from the authors upon reasonable request.

Supplemental document. See Supplement 1 for supporting content.

\section{References}

[1] M. Christensen, A. K. Hansen, D. Noordegraaf, P. M. W. Skovgaard, and O. B. Jensen, "Second-harmonicgeneration-based technique for examining laser diode wavelength dynamics in the $\mu$ s to ms range," Appl. Opt., vol. 57, no. 6, p. 1432, 2018, doi: 10.1364/AO.57.001432.

[2] P. Albrodt et al., "Coherent beam combining of high power quasi continuous wave tapered amplifiers," Opt. Express, vol. 27, no. 20, p. 27891, Sep. 2019, doi: 10.1364/OE.27.027891.

[3] J. W. Goodman, "Some fundamental properties of speckle*," J. Opt. Soc. Am., vol. 66, no. 11, p. 1145, Nov. 1976, doi: 10.1364/JOSA.66.001145.

[4] J. W. Goodman, "Statistical Properties of Laser Speckle Patterns," in Laser Speckle and Related Phenomena, vol. 6, 1975, pp. 9-75.

[5] H. T. Yura, B. Rose, and S. G. Hanson, "Dynamic laser speckle in complex ABCD optical systems," $J$. Opt. Soc. Am. A, vol. 15, no. 5, p. 1160, May 1998, doi: 10.1364/JOSAA.15.001160.

[6] H. Cao, "Perspective on speckle spectrometers," J. Opt., vol. 19, no. 6, p. 060402, Jun. 2017, doi: $10.1088 / 2040-8986 /$ aa7251.

[7] N. H. Wan, F. Meng, T. Schröder, R.-J. Shiue, E. H. Chen, and D. Englund, "High-resolution optical spectroscopy using multimode interference in a compact tapered fibre," Nat. Commun., vol. 6, no. 1, p. 7762, Nov. 2015, doi: $10.1038 /$ ncomms8762.

[8] N. K. Metzger et al., "Harnessing speckle for a sub-femtometre resolved broadband wavemeter and laser stabilization," Nat. Commun., vol. 8, no. 1, p. 15610, Aug. 2017, doi: 10.1038/ncomms15610.

[9] Y. Wan, S. Wang, X. Fan, Z. Zhang, and Z. He, "High-resolution wavemeter using Rayleigh speckle 
obtained by optical time domain reflectometry," Opt. Lett., vol. 45, no. 4, p. 799, Feb. 2020, doi: 10.1364/OL.384248.

[10] M. Chakrabarti, M. L. Jakobsen, and S. G. Hanson, "Speckle-based spectrometer," Opt. Lett., vol. 40, no. 14, p. 3264, Jul. 2015, doi: 10.1364/OL.40.003264.

[11] M. L. Jakobsen, H. T. Yura, and S. G. Hanson, "Speckles and their dynamics for structured target illumination: optical spatial filtering velocimetry," J. Opt. A Pure Appl. Opt., vol. 11, no. 5, p. 054001, May 2009, doi: 10.1088/1464-4258/11/5/054001.

[12] S. G. Hanson, M. L. Jakobsen, and M. Chakrabarti, "The dynamic speckle-based wavemeter," in Speckle 2018: VII International Conference on Speckle Metrology, Sep. 2018, no. September 2018, p. 115, doi: 10.1117/12.2319873.

[13] M. Sjödahl, “Accuracy in electronic speckle photography,” Appl. Opt., vol. 36, no. 13, p. 2875, May 1997, doi: $10.1364 / A O .36 .002875$.

[14] M. Sjödahl and L. R. Benckert, "Systematic and random errors in electronic speckle photography," Appl. Opt., vol. 33, no. 31, p. 7461, Nov. 1994, doi: 10.1364/AO.33.007461.

[15] S. C. Schneider, S. J. Rupitsch, and B. G. Zagar, "Signal Processing for Laser-Speckle Strain-Measurement Techniques," IEEE Trans. Instrum. Meas., vol. 56, no. 6, pp. 2681-2687, Dec. 2007, doi:

10.1109/TIM.2007.908251.

[16] K.-H. Hasler et al., "5-W DBR Tapered Lasers Emitting at $1060 \mathrm{~nm}$ With a Narrow Spectral Linewidth and a Nearly Diffraction.Limited Beam Quality," IEEE Photonics Technology Letters, vol. 20, no. 19, p. 1648, Oct. 2008 